\title{
The Application of Online Consultant Mode in Postpartum Maternity and Infant at Home in COVID-19 Pandemic
}

\author{
Zhili Chen, Lin Wang*, Jiali Chen \\ Transitional Care Department, the First Affiliated Hospital of Jinan University, Guangzhou, China \\ Email address: \\ chenzl1215@sina.com (Zhili Chen),wanglin18903078509@126.com (Lin Wang) \\ ${ }^{*}$ Corresponding author
}

To cite this article:

Zhili Chen, Lin Wang, Jiali Chen. The Application of Online Consultant Mode in Postpartum Maternity and Infant at Home in COVID-19

Pandemic. American Journal of Nursing Science. Vol. 9, No. 4, 2020, pp. 231-234. doi: 10.11648/j.ajns.20200904.21

Received: May 23, 2020; Accepted: June 8, 2020; Published: June 20, 2020

\begin{abstract}
Objective: To explore the effects of providing guidance postpartum maternity and infant at home by online consultation mode under the situation of COVID-19 pandemic. Methods: The International Board Certified Lactation Consultants (IBCLC) developed the procedure of online consultation for the maternity and infant during the COVID-19 pandemic, used WeChat mini program to provide health education and guidance for postpartum maternity and their families, and monitored their conditions through the follow-up visits. Results: During the pandemic period of COVID-19 from January $20^{\text {th }}$ to April $30^{\text {th }}, 2020$, Transitional Care Department of the authors' hospital used the WeChat mini program to provide health education and guidance for a total of 105 cases of patients, of which 8 cases were advised to consult relevant specialties online and 8 cases were recommended to visit hospital. The remaining 89 cases of patients had their medical problems solved effectively through a total of 177 times of online counseling. The satisfaction of maternity and their families on the online consulting service reached $98.63 \%$. Conclusion: Under the situation of the COVID-19 pandemic, the application of the online consultant mode meets the medical needs of the maternity and infant at home; avoids the risk of cross-infection caused by face-to-face communication; and ensure the health of the patients and their families.
\end{abstract}

Keywords: COVID-19, Online Consultation Mode, Maternity and Infant at Home

\section{Introduction}

In December 2019, various cases of novel coronavirus pneumonia arose in China [1-2]. A global novel coronavirus pneumonia pandemic spread rapidly. On January 20th, 2020, the National Health Committee of China subsumed the novel coronavirus pneumonia into Category B infectious substance and managed it based on the preventions and control measures of Category A infectious diseases [3]. On February 2nd, 2020, the notice on the prevention and control of pneumonia in children and mothers with novel coronavirus infections issued by Department of Maternal and Child Health of China pointed out that the children and the maternity were vulnerable to the COVID-19. In order to further improve the prevention and control measures of the COVID-19, Chinese primary health care institutions suspended face-to-face visits to infant and children during the pandemic, conducting online consultation and guidance through WeChat, telephone, video instead [4]. The Transitional Care Department of the authors' hospital actively responded to the government's policy, adopting the mode of Internet consulting services to provide online consultation service for the maternities and infants at home. This measure achieved a pleasant result.

\section{Methods}

\subsection{Participants}

The maternity and infant who were served by the Transitional Care Department of the authors' hospital through the online consultation service during the COVID-19 pandemic from January 20th to April 30th, 2020 were selected for this study.

\subsection{Procedure}

\subsubsection{Establishment of an Online Consultation Platform Using WeChat Mini Program}

The Wechat mini program was established in 2019 and online consulting function was improved in January, 2020. 
The WeChat mini program allows patients to make appointments online and to upload case information in a variety of ways such as text descriptions, uploading pictures, and audio descriptions. Medical staff can also use these ways to provide consulting services to patients.

\subsubsection{Development of the Procedure of Online Consultation for the Maternity and Infant During the COVID-19 Pandemic}

The online consultation and follow-up services for the patients who have applied for consultation through the WeChat mini program were conducted by the International Board Certified Lactation Consultants (IBCLC) of Transitional Care Department. The processes of online consultation are as followed: (1) Upon receipt of a request for online consultation from patients, IBCLC establishes a consultation file and responds to the patients within 12 hours. According to the requirements of the appointment service, the sequence of the consultation is planned based on the priority of the questions asked by the patients. For instance, the priority of the online consultation can be given for mastitis during the time for breastfeeding, breast swelling, having fever, etc. (2) IBCLC Conducts an online assessment to gather relevant information to identify nursing care issues. IBCLC communicates with the patients online to understand their needs and ask the patients to refine the information related to the content of the consultation, which can be completed in the form of text, picture, or video. (3) IBCLC provides counseling services based on assessment questions. On the day of consultation, patients will be reminded of the consultation time 10 minutes in advance on WeChat. The consultation is operated in the form of text, voice message, and pictures. Voice call, video calls, and other media functions will be used if necessary. Patients are guided on how to solve problems and skills of care. If IBCLC encounters problems that do not fall within their scope of specialties during the process of communication, they will provide a referral to the corresponding specialty. IBCLC is required to respond to the patient's feelings, pay attention to the patient's psychological changes, and empathize with the patient in the consultation process. (4) After the consultation, the patients are asked to repeat the main points of the current problem. The plan for the patients is adjusted according to the actual situation of the patients and their family. Patients are also instructed on protective measures at home under the situation of the COVID-19 pandemic. (5) Online follow-up visits are provided in two days after the consultation, which mainly includes the evaluation of the difficulty of the plan as well as the result of the plan.

\subsection{Evaluation Index}

(1) Maternities and infants Cases who were provided the online consultation service from January 20th to April 30th, 2020 were collected; (2) Monitoring patients' situations through the follow-up visits. For example, whether the patients acquire the knowledge and skills; whether their symptoms getting improved; and whether the patients visit the hospital. (3) The satisfaction of the patients and their families following the provision of online counseling services.

\section{Result}

A total of 105 patients were served by IBCLC through online consultation from January 20th to April 30th, 2020, of which 8 cases were asked to the corresponding specialties and 8 cases were recommended to visit the hospital. The service content is mainly divided into two categories: maternity problems such as lactation mastitis, inadequate breast milk, stagnant breast milk, nipple pain, etc.; common problems for infants such as eczema, flatulence care, umbilical cord care, jaundice observation and care, etc. Service items and cases were shown in table 1.

A total of 177 times of counseling of maternity and infant problems were solved for 89 patients. By providing the online consultation and online follow-up visits, the maternities and their family members could acquire the knowledge and skills and solve their problems properly. None of the maternities ant their babies visited the hospital. The detail of the results was illustrated in table 1 .

The satisfaction of maternitiess and their family members with the online consultant service was $98.63 \%$.

Table 1. Service items and service cases of online consultant mode for maternity and infantin the COVID-19 pandemic.

\begin{tabular}{lll}
\hline Object & Service Items & Cases (n, \%) \\
\hline Maternities & Breastfeeding instruction & $58(32.77)$ \\
& Nursing guidance of nipples pain & $18(10.17)$ \\
& Nursing guidance of breast swelling & $14(7.91)$ \\
and pain & $8(4.52)$ \\
& Weaning guidance & $1(0.56)$ \\
& Oral medicine administration & $1(0.56)$ \\
Infants & Postpartum psychological nursing & $14(7.91)$ \\
& Nursing care of jaundice & $14(7.91)$ \\
& Flatulence nursing care & $11(6.22)$ \\
& Skin and mucous membrane care & $9(5.09)$ \\
& Umbilical care & $8(4.52)$ \\
& Instruction of Crying placatory & $6(3.39)$ \\
& Instruction of Vaccination & $5(2.82)$ \\
& Excretion care & $3(1.70)$ \\
& Eczema care & $2(1.13)$ \\
& Oral medicine administration & $5(2.82)$ \\
& others & $177(100.00)$ \\
\hline
\end{tabular}

\section{Discussion}

\subsection{The Medical Needs of Maternity and Infant at Home Could Be Addressed Through the Online Counseling Services Under the COVID-19 Pandemic Situation}

With the development of obstetrics technology, the hospitalization time of maternity and newborn has shortened, resulting in limited knowledge and skills of the postnatal home-care for maternities and their families to learn during their hospitalization [5]. Due to inexperience in self-care and caring for the baby, the maternities will encounter many 
problems that can affect the health of them and infants after discharge. Although maternities can access relevant information using the Internet, it is difficult for them to distinguish the authenticity of the information found online. Therefore, the maternities and their family still need professional guidance and assistance after discharge [6]. The authors' hospital has been providing postnatal extensional care services since 2007. The working mode of home-care service combined with online consultation is to carry out for maternities and infants after discharge from the hospital. However, due to the situation of the COVID-19 pandemic, reducing the risk of cross-infection caused by face-to-face communication is necessary. As a result, we adjusted the nursing service mode during the COVID-19 pandemic period, mainly using internet technology to perform the online consultation. The practice of online consultant mode presented that the online consultant services could minimize the risk of infection caused by face-to-face visits. The maternities and infants can get timely and individualized guidance from medical professionals at home. It can not only effectively ensure the maternities and infants health as well as satisfaction, but also contribute to the prevention and control of pandemic.

\subsection{Online Consultant Services Are More Adaptable to the Maternity and Infant}

The results of online consultation service also showed that the main problems for the maternity and infant during the pandemic were infant diseases such as eczema and flatulence, jaundice; the outstanding problems of maternities such as mastitis during lactation, painful nipple cracking and stagnant breast milk. These results were consistent with the findings of $\mathrm{Li}$, Fan, Zou and $\mathrm{Wu}$ [7-10]. With the development of the economy and society and the popularity of information technology, the Internet is widely used in various fields such as education, medical care, and enterprise More and more people are willing to experience the convenience and intelligence brought by the 'Internet Plus' services. The main communication application used in this study is WeChat mini program which is a free live chatting application with a large user base. It takes into account both the travel burden and economic pressure of the maternity and infant. It has been widely applied in clinical nursing care [11-13]. The study indicated that the younger population is more receptive to new things and more willing to accessing health care service resources through the Internet than the elderly population $[14,15]$. The postnatal population is a group of young people with certain reading, writing, and information comprehension abilities, who can skillfully apply the intelligent network system and utilize video, picture and text for information exchange. Accordingly, the maternities are proactive in obtaining medical knowledge and skills through the online consultant, and the counseling issues are solved efficiently.

\section{Conclusion}

Online consultant services play a crucial role to meet the medical care needs of the maternity and infant under the COVID-19 pandemic situation due to its advantages. They can directly get professional guidance and assistance at home, and their self-care ability can also be improved. The risk of cross-infection is also reduced. Furthermore, the mode of consultant services avoids the disconnect of postnatal services after discharge from the hospital. Overall, the online consultant services mode is worth to popularize and apply in more fields.

\section{References}

[1] Huang C., Wang Y. and Li X. (2020). Clinical features of patients infected with 2019 novel coronavirus in Wuhan, China [J]. Lancet, 395 (10223): 497-506.

[2] Wang C, Horby P. W., Hayden F. G. (2020). A novel coronavirus outbreak of global health concern. Lancet, 395 (10223): 470-473.

[3] The Central People's Government of the People's Republic of China. (2020). Novel coronavirus pneumonia is included in the statutory management of infectious diseases. http://www.gov.cn/xinwen/2020-01/21/content_5471153.htm.

[4] Department of Maternal and Child Health. (2020). The notice on the prevention and control of pneumonia caused by novel coronavirus in children and pregnant patients. http://www.nhc.gov.cn/fys/s7902/202002/de2d62a5711c41ef9 b2c4b6f4d1f2136.shtml.

[5] Wang Y. (2017). The application of transitional care in improving maternity self-care ability and quality of life after discharge. Contemporary Nurse, (12): 95-97.

[6] Yonemoto N, Dowswell T, Nagai S, et al. (2017). Schedules for home visits in the early postpartum period [J]. Cochrane Database Systematic Reviews, 8: Cd009326.

[7] Li L. D., Xu X. N. and Tan Q. X. (2017). Investigation and analysis of community postpartum women's demand for health knowledge. Chinese Medical Science, 7 (04): 51-54.

[8] Fan Z. C. (2014). The problems and countermeasures of postpartum visit. Contemporary Nurse, (05): 27-29.

[9] Zou L., Zheng Y. N. and Wei S. H. (2020). Internet plus nursing practices promote the effects of exclusive breastfeeding. Southeast National Defense Medicine, 2020, 22 (01): 85-87.

[10] Wu Z. H. (2019). Application effect of "Internet plus transitional care" in nursing guidance of discharged patients in obstetrics department. Horizon of Science and Technology, (24): 209-210.

[11] Gao Q., Xu X. J. and Zhou C. C. (2019). Construction and implementation of "Internet + " puerperal nursing follow-up system. Nursing Research, 33 (02): 340-343.

[12] Yin X. F. and Zhang Q. (2018). The influence of "online school for pregnant women" on improving the self-care ability of pregnant patients. Chinese Community Physician, 34 (28): $162-163+165$.

[13] Tang M. Y. and Li X. L. (2018). Advances in the application of mobile applications in disease transitional care. Chinese Journal of Nursing. 53 (03): 363-365. 
[14] James DCS and Harville C II. (2017). Barriers and motivators to participating in mHealth research among African Americans men. American Journal of Mens Health, 11 (6): 1605-1613.
[15] Tian L. and Chen Y. (2019).'Internet plus" transitional care nursing research progress. Journal of Nursing Science, 34 (17): 17-20. 